\title{
Pancyclicity and Cayley graphs on generalized dihedral groups
}

\author{
Brian Alspach and Alexander Muir* \\ This paper is dedicated to Adrian Bondy on the occasion of his seventieth \\ birthday
}

\begin{abstract}
We investigate the existence of cycles of various lengths in connected Cayley graphs of valency at least 3 on generalized dihedral groups.
\end{abstract}

AMS 2000 SUBJECT ClASSifications: 05C25, 05 C38.

KeYwORds AND PHRASEs: Pancyclic, girth, odd girth, Cayley graph, generalized dihedral group.

\section{Introduction}

Graphs are undirected, have no loops and no multiple edges throughout this paper. Cycles in graphs have been studied extensively from the early days of the subject. A graph $X$ is pancyclic if it contains cycles of every possible length from 3 through the order of $X$. This basic concept was introduced by Bondy in [6] making the topic especially relevant for an issue dedicated to him. If every vertex is in cycles of every possible length, we say $X$ is vertex-pancyclic, and if the same is true for every edge, then we say that $X$ is edge-pancyclic.

The girth of a graph $X$, denoted $\gamma(X)$, is the length of a shortest cycle in $X$. The odd girth of a graph $X$, denoted $\gamma_{o}(X)$, is the length of a shortest cycle of odd length in $X$. If $X$ is bipartite, we say the odd girth of $X$ is undefined. Thus, when we write that $X$ has some odd girth, we are assuming that $X$ actually has odd length cycles.

A graph cannot be pancyclic unless it has girth 3 and yet it makes sense to consider graphs with larger girth with respect to richness in the possession of cycles of various lengths. To this end, a bipartite graph $X$ is even pancyclic if $X$ contains cycles of all even lengths from 4 through $2\lfloor n / 2\rfloor$, where $n$ is the order of $X$. The terms even vertex-pancyclic and even edge-panyclic

${ }^{*}$ This work was done during an Undergraduate Summer Research Scholarship at the University of Newcastle. 
have the obvious meanings. If a graph $X$ has girth $\gamma$, then it is called weakly pancyclic when it has cycles of all lengths from $\gamma$ through the circumference of $X$. The terms weakly vertex-pancyclic and weakly edge-pancyclic have the obvious meanings. Finally, an important concept we consider is weakly odd pancyclic which means the graph has odd length cycles of all lengths of the form $\gamma_{o}(X)+2 k$ as long as $\gamma_{o}(X)+2 k$ is less than or equal to the circumference of $X$.

Vertex-transitive graphs serve as models for many applications of graphs. Cayley graphs are a rich source of vertex-transitive graphs and have been studied widely with respect to many properties of graphs. Some references for pancyclicity and Cayley graphs are $[4,5,7,9,10]$. The cited papers all studied subclasses of either circulant graphs or $n$-dimensional cubes. The following theorem [2] then considerably generalized that work by completely describing what happens for Cayley graphs on abelian groups.

Theorem 1.1. Let $X$ be a connected Cayley graph of valency at least 3 on an abelian group. The following statements hold for $X$ :

1. $X$ is even edge-pancyclic;

2. if $X$ is not bipartite, then $X$ is weakly odd vertex-pancyclic; and

3. if $X$ is not bipartite, then every edge of $X$ is contained in cycles of lengths $\gamma_{o}(X)+2 k$ for all $k$ satisfying $k>0$ and $\gamma_{o}(X)+2 k \leq|V(X)|$.

The preceding theorem provides strong impetus for examining the possibility of achieving similar results for other families of Cayley graphs. We consider the family of Cayley graphs on generalized dihedral groups in this paper.

\section{Describing the Cayley graphs}

In order to exploit Theorem 1.1, we want to consider groups that are 'almost abelian' in some sense. The family of generalized dihedral groups is such a family and that is what we now study. The definition follows. Given an abelian group $H$, the generalized dihedral group $D_{H}$ contains $H$ as a subgroup of index 2 and an involution $\tau \notin H$ conjugating every element of $H$ to its inverse, that is, $\tau g \tau=g^{-1}$ for all $g \in H$. It is easy to see that $\left|D_{H}\right|=2|H|$, and every element of $D_{H}$ that is not in $H$ may be written as $h \tau$ for some $h \in H$.

In order to clarify our terminology, here is the definition of a Cayley graph we are using. Given a group $G$, let $S \subset G$ such that $1 \notin S$ and $S=S^{-1}$. The Cayley graph $\operatorname{Cay}(G ; S)$ has its vertices labelled bijectively with the elements of $G$ and there is an edge joining the vertex labelled $g$ to 
all vertices whose labels have the form $g s$ as $s$ runs through $S$ and $g$ runs through $G$. Note that the edge joining $g$ to $g s$ is the same edge joining $g s$ to $\mathrm{gss}^{-1}=g$. Also note that we use multiplicative notation for the group operation including abelian groups. The set $S$ is called the connection set for $\operatorname{Cay}(G ; S)$.

We want to take a look at the structure of Cayley graphs on generalized dihedral groups because there are properties they possess that help subsequent work. The remainder of this section consists of five useful lemmas and their proofs.

Let $X=\operatorname{Cay}\left(D_{H} ; S\right)$. The first feature we notice is that there is a natural partition of the connection set $S$. We define $S_{1}=S \cap H$ and $S_{2}=S-S_{1}$.

The subgroup $H$ has index 2 in $D_{H}$ so that $H$ is a normal subgroup. We designate the two cosets of $H$ as $H$ and $H \tau$. It is easy to see that any $s \in S_{2}$ is an involution and generates a perfect matching joining the vertices of $H$ to the vertices of $H \tau$. Thus, if $S_{2}=\emptyset$, the graph $X$ is disconnected so that the assumption that $X$ is connected implies that $S_{2} \neq \emptyset$.

If $s \in S_{1}$, then there is an edge joining $g$ and $g s$ for all $g \in H$. If we take any involution $h \tau$ in the right coset $H \tau$, then because $s^{-1} \in S$, there is an edge joining $g(h \tau)$ and $g s(h \tau)=g(h \tau) s^{-1}$. That is, right multiplication by $h \tau$ is an isomorphism between the subgraph $X_{1}$ induced on $H$ and the subgraph $X_{2}$ induced on $H \tau$. This fact is encapsulated as the first of the four lemmas.

Lemma 2.1. Let $X=\operatorname{Cay}\left(D_{H} ; S\right)$ be a Cayley graph on the generalized dihedral group $D_{H}$. If $X_{1}$ is the subgraph induced by $X$ on $H$ and $X_{2}$ is the subgraph induced by $X$ on $H \tau$, then right multiplication by $h \tau, h \in H$, is an isomorphism from $X_{1}$ onto $X_{2}$.

The following lemma also proves to be useful in proving the main result, but its proof requires the following definition. Given positive integers $m, n, \ell$, where $n \geq 4$ is even and $m+\ell$ is even, define the honeycomb toroidal graph $\operatorname{HTG}(m, n, \ell)$ as follows. It has vertices $u_{i, j}$, for $0 \leq i \leq m-1$ and $0 \leq j \leq n-1$, and the following edges. The vertex $u_{i, j}$ is adjacent to $u_{i, j \pm 1}$ for all $j$ with subscript arithmetic carried out modulo $n$. There is an edge from $u_{i, j}$ to $u_{i+1, j}$ whenever $i<m-1$ and $i+j$ is odd. In addition, there is an edge from $u_{m-1, j}$ to $u_{0, j+\ell}$ for $m+j$ even. The honeycomb toroidal graph $\operatorname{HTG}(4,6,4)$ is shown in Figure 1.

Lemma 2.2. Let $X=\operatorname{Cay}\left(D_{H} ; S\right)$ be a Cayley graph on the generalized dihedral group $D_{H}$. If $X$ is connected, $S_{1}=\emptyset$ and $s$ is an arbitrary element of $S_{2}$, then $X$ has a Hamilton path such that the terminal vertex in $H$ is incident with an edge generated by $s$. 


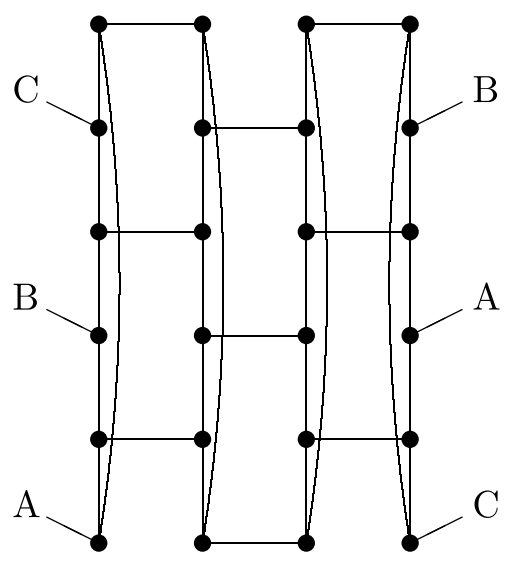

Figure 1.

Proof. A Hamilton path in $X$ must have one terminal vertex in $H$ and the other terminal vertex in $H \tau$ because $X$ is bipartite and $|H|=|H \tau|$. This means the edge incident with the terminal vertex in $H$ is uniquely determined. Because each element of $S_{2}$ generates a perfect matching joining vertices of $H$ with vertices of $H \tau,\left|S_{2}\right| \geq 2$ because $X$ is connected. We proceed by building the required Hamilton path recursively.

Choose the fixed element $s \in S_{2}$ and some other element $s_{1} \in S_{2}$. If the 2 -factor generated by $s, s_{1}$ is a Hamilton cycle, we are done because deleting any edge generated by $s_{1}$ leaves a Hamilton path such that the terminal vertex in $H$ is incident with an edge generated by $s$. Otherwise, the 2-factor is composed of cycles of the same even length $r$. Because $X$ is connected, there is an $s_{2} \in S$ such that $s_{2}$ does not belong to the group $\left\langle s, s_{1}\right\rangle$ generated by $s$ and $s_{1}$.

If an edge generated by $s_{2}$ joins a vertex of $H \tau$ in some $r$-cycle $C_{1}$ to a vertex of $H$ in another $r$-cycle $C_{2}$, then $s_{2}$ generates a perfect matching between the vertices of $C_{1} \cap H \tau$ and the vertices of $C_{2} \cap H$ because of Lemma 2.1. So we define an auxiliary digraph $\vec{Y}$ whose vertices are the $r$ cycles generated by $s, s_{1}$, with an arc from the $r$-cycle $C$ to the $r$-cycle $C^{\prime}$ in $\vec{Y}$ when $s_{2}$ generates a perfect matching joining the the vertices of $C \cap H \tau$ to the vertices of $C^{\prime} \cap H$.

The outvalency and invalency of each vertex in $\vec{Y}$ is 1 , that is, each component is a directed cycle. So the graph generated by $s, s_{1}, s_{2}$ corresponding to one of the directed cycles is a honeycomb toroidal graph $\operatorname{HTG}(m, r, \ell)$, where the $r$-cycles form the columns (see Figure 1). The edges joining the $r$-cycles in a cyclic fashion are generated by $s_{2}$. It is easy to see that there is a Hamilton path starting at any vertex and using either column edge as 
the first edge. Thus, if this graph spans $X$, we are done because the edges of the columns are generated by $s$ and $s_{1}$.

If the honeycomb toroidal graph is not all of $X$, then the three involutions $s, s_{1}, s_{2}$ generate components that are isomorphic copies of the same honeycomb toroidal graph. Because $X$ is connected, there must be an involution $s_{3} \in S_{2}$ that joins different components together in a cyclic fashion, and $s_{3}$ generates a perfect matching joining the vertices of a component's intersection with $H \tau$ to the vertices of another component's intersection with $H$. The resulting subgraph then has a spanning path starting at any vertex whose first edge is generated by either $s$ or $s_{1}$. We continue adding elements of $S_{2}$ until we reach a connected spanning subgraph of $X$ and the conclusion follows.

The subgraph $X_{1}$ plays a significant role in this work. There are two attributes of this subgraph that are germane to the discussion. The first is whether or not $X_{1}$ is connected and the second is its valency. For example, if $X_{1}$ is connected and has valency at least 3, then we may bring the full power of Theorem 1.1 into play. These two attributes determine the cases that arise in the proof of Theorem 3.1.

Another subgraph that plays an important role is the following. Let $X=\operatorname{Cay}\left(D_{H} ; S\right)$ be connected and let $X_{1}, X_{2}, S_{1}, S_{2}$ be as before. Choose any element $s \in S_{2}$. Let $Y(s)$ denote the subgraph of $X$ composed of $X_{1} \cup X_{2}$ together with edges of the perfect matching generated by $s$.

Lemma 2.3. If $X=\operatorname{Cay}\left(D_{H} ; S\right)$ is connected and $s^{\prime}$ is any element of $S_{2}$, then $Y\left(s^{\prime}\right)$ is isomorphic to a Cayley graph on an abelian group.

Proof. Let $G$ be the abelian group $H \times Z_{2}$, where we use $Z_{2}=\{1,-1\}$. Let the connection set $T$ for $G$ be $\left\{(s, 1): s \in S_{1}\right\} \cup\{(1,-1)\}$. Define the mapping $\varphi$ from $Y$ to $\operatorname{Cay}(G ; T)$ by $\varphi(h)=(h, 1)$ and $\varphi(h s)=(h,-1)$. It is easy to verify that this is an isomorphism from which the result follows.

An important modification we employ frequently is the replacement of an edge $u v$ by a path of length 3 joining $u$ and $v$. We call the 3 -path a bypass. We sometimes specify the 3-path via the vertices along the path and use the notation $\left(u, w_{1}, w_{2}, v\right)$-bypass. Alternatively, we sometimes specify the 3 -path via the elements generating the successive edges. So if the sequence $s_{1}, s_{2}, s_{3}$ generates a 3 -path from $u$ to $v$, then we use the notation $\left(s_{1}, s_{2}, s_{3}\right)$ bypass.

Lemma 2.4. Let $X=\operatorname{Cay}\left(D_{H} ; S\right)$ be connected of order $2 m$ and let $X_{1}$ be connected and have valency at least 3. If $C$ is a cycle of length $\ell$ in $X$ using exactly one edge from $X_{1}$ or exactly one edge of $X_{2}$, then we can find cycles 
of lengths $\ell+2 k$ intersecting $X_{1}$ or $X_{2}$, respectively, in a path of length $2 k+1$ whenever $2 k+1<m$.

Proof. Let $C$ be a cycle of length $\ell$ intersecting $X_{1}$ in the edge $u v$. Because $X_{1}$ is a connected Cayley graph on an abelian group and has valency at least 3, Theorem 1.1 part 1 implies that $u v$ is contained in cycles of all even lengths from 4 through $2\lfloor m / 2\rfloor$. We may then replace the edge $u v$ in $C$ with odd length paths in $X_{1}$ to get the desired result. The identical proof works when $C$ intersects $X_{2}$ in a single edge.

Note that $2\lceil m / 2\rceil-1$ is the largest odd integer less than or equal to $m$.

Lemma 2.5. Let $X=\operatorname{Cay}\left(D_{H} ; S\right)$ be connected of order $2 m$ and let $X_{1}$ be connected and have valency at least 2. If $X$ is not bipartite, then $X$ has cycles of all odd lengths from $2\lceil m / 2\rceil-1$ through $2 m-1$.

Proof. If $X_{1}$ is not bipartite, then choose $s^{\prime} \in S_{2}$. The subgraph $Y\left(s^{\prime}\right)$ is not bipartite, has valency at least 3 and is a Cayley graph on an abelian group by Lemma 2.3. So it has cycles of the required odd lengths by Theorem 1.1 part 2 because the odd girth of $Y\left(s^{\prime}\right)$ is at most $m$.

If $m$ is even and $X_{1}$ is bipartite, then let $C=u_{1} u_{2} \cdots u_{m}$ be a cycle that spans $X_{1}$. Denote the parts of the bipartition of $X_{1}$ as $A$ and $B$. Without loss of generality, let $A$ contain the vertices $u_{i}$ with odd subscript and $\mathrm{B}$ contain those with even subscript.

Let $s_{1} \in S_{2}$ and using Lemma 2.1, we know that right multiplication by $s_{1}$ gives us a copy of $C$, say $C^{\prime}$, in $X_{2}$, where the two copies are joined by the perfect matching generated by $s_{1}$. For ease of notation, let $u_{i}^{\prime}=u_{i} s_{1}$ for all $i$. The trivalent subgraph formed by $C, C^{\prime}$ and the perfect matching joining them is isomorphic to the cartesian product $C \square K_{2}$ which is bipartite. Hence, the bipartition of $C^{\prime}$ is determined.

Because $X$ itself is not bipartite, there must be an $s_{2} \in S_{2}$ that does not respect the bipartition. Let $u_{1} u_{j}^{\prime}$ be an edge generated by $s_{2}$ so that $j$ is even. The vertices $u_{1}^{\prime}$ and $u_{j}^{\prime}$ partition the cycle $C^{\prime}$ into two odd length subpaths one of which has length at most $m / 2$. Without loss of generality, let the subpath $u_{1}^{\prime} u_{2}^{\prime} \cdots u_{j}^{\prime}$ have length at most $m / 2$.

Then $u_{1} u_{1}^{\prime} u_{2}^{\prime} \cdots u_{j}^{\prime} u_{1}$ is an odd length cycle $C(0)$. Because of the cartesian product subgraph, we know that $u_{1} u_{m} u_{m}^{\prime} u_{1}^{\prime}$ is a bypass on the edge $u_{1} u_{1}^{\prime}$ in $C(0)$. Next we can take the bypass $u_{m} u_{m-1} u_{m-1}^{\prime} u_{m}^{\prime}$ on the newly added edge $u_{m} u_{m}^{\prime}$. We may continue this way building longer and longer odd length cycles until we reach a cycle $C(1)$ that uses all the vertices of $X_{2}$.

If only one vertex of $C$ does not appear in $C(1)$, then we are done. Otherwise, choose a matching along the subpath of $C$ induced by the vertices 
of $C$ not appearing in $C(1)$. We then use these matching edges and edges generated by $s_{1}$ to form bypasses on edges of $C^{\prime}$ appearing in $C(1)$. We obtain all desired odd lengths.

\section{Main result}

Theorem 1.1 provides a complete answer for connected Cayley graphs on abelian groups, whereas, for connected Cayley graphs on generalized dihedral groups, the next theorem provides a complete answer only when $S_{1} \neq \emptyset$. This is the main result in the paper. Its proof is followed by a brief discussion of the case that $S_{1}=\emptyset$.

Theorem 3.1. Let $D_{H}$ be a generalized dihedral group, $X=\operatorname{Cay}\left(D_{H} ; S\right)$, $|S| \geq 3, S_{1}=S \cap H, S_{2}=S-S_{1}$ and $X$ be connected. The following statements hold for $X$ :

1. if $S_{1} \neq \emptyset$, then $X$ is even edge-pancyclic; and

2. if $S_{1} \neq \emptyset$ and $X$ is not bipartite, then $X$ is weakly odd vertex-pancyclic.

Proof. We now embark on a proof of Theorem 3.1. Here is an outline of the proof. There are two basic cases: $X_{1}$ is connected and $X_{1}$ is not connected, where we recall that $X_{1}$ denotes the subgraph induced on $H$ by $X$. We first take care of the case that $X_{1}$ is connected. It is a direct argument and proceeds according to the valency of $X_{1}$.

When $X_{1}$ is not connected, we take a contraction and employ the fact that the contraction contains a Hamilton path. We then use the contraction to obtain a useful spanning subgraph of the Cayley graph.

\subsection{Connected case}

Let $X=\operatorname{Cay}\left(D_{H} ; S\right)$. This subsection deals exclusively with the case that $X_{1}$ is connected. When referring to edges of $X$, we call edges contained in either $X_{1}$ or $X_{2}$ internal edges, and we call edges with one end vertex in $H$ and the other end vertex in $H \tau$ linking edges.

If the valency of $X_{1}$ is 1 , then $X_{1}=K_{2}$ and $X$ has order 4 . As $|S| \geq 3$, $X=K_{4}$ which is pancyclic and every edge lies in cycles of lengths 3 and 4 . This exhausts the possibilities when $X_{1}$ has valency 1 .

We now proceed to the case that $X_{1}$ has valency at least 2 . When $X_{1}$ is connected, has valency 2 and is not cyclic, then $H$ is isomorphic to $Z_{2} \times Z_{2}$, with $S_{1}$ consisting of two involutions. The group $D_{H}$ is abelian because every non-identity element of the group has order 2 . The conclusion follows from Theorem 1.1 in this case. So we assume that $H$ is cyclic when the valency is 2 . 
If we choose any $s_{1} \in S_{2}$, then $Y\left(s_{1}\right)$ is even edge-pancyclic by Lemma 2.3 and Theorem 1.1. Thus, $X$ itself is even edge-pancyclic. So this completes the argument when $X$ is bipartite.

If $X$ is not bipartite, we are going to prove the following statement by induction on the number of linking edges: If $C$ is a cycle of odd length $\ell$ containing exactly $2 t$ linking edges, then $X$ has cycles of all odd lengths from $\ell$ through $2 m-1$.

Suppose first that $C$ has no linking edges. When $X_{1}$ has valency 2 , then $\ell=m$ must hold. When $X_{1}$ has valency bigger than 2 , then $X_{1}$ contains odd length cycles of lengths $\ell$ through the odd part of $m$ by Theorem 1.1. In both cases, Lemma 2.5 gives us the rest of the odd lengths through $2 m-1$.

We cannot use no linking edges as the base for induction so that we must argue two linking edges separately. So we now suppose that $C$ has precisely two linking edges. Because of Lemma 2.5, we may assume $\ell<2\lceil m / 2\rceil-1$, and it suffices to find cycles of all odd lengths from $\ell$ through $2\lceil m / 2\rceil-3$.

The cycle $C$ is composed of a subpath $P=u_{1} u_{2} \cdots u_{j}$ of $X_{1}$, a subpath $Q=v_{1} v_{2} \cdots v_{k}$ of $X_{2}$ and the two linking edges $u_{1} v_{1}$ and $u_{j} v_{k}$. Without loss of generality, assume $j<k$ (the lengths must be different because $\ell$ is odd). If $j=1$, that is, $P$ has length 0 , then let $s \in S_{1}$ and $s_{1} \in S_{2}$ generate the edge joining $u_{1}$ and $v_{1}$. Consider the $\left(s, s_{1}, s\right)$-bypass on the edge $u_{1} v_{1}$, where $x=u_{1} s s_{1} s$. If $x$ is not on $C$, then we obtain a cycle of length $\ell+2$ with a single edge in $X_{1}$. If $x$ is on $C$, then we obtain a cycle of odd length $\ell^{\prime} \leq \ell$ with a single edge in $X_{1}$.

If $j>2$, that is, $P$ has length at least 2, let $s$ generate the edge from $u_{1}$ to $u_{2}$. Let $x=u_{2} s_{1}$. If $x$ is on $C$, then the edge $u_{2} x$ is a chord of $C$ and we obtain either an odd length cycle with one edge in $X_{1}$ and two linking edges, or an odd length cycle such that the path in $X_{1}$ has length $j-2$ and there are two linking edges. Both cycles have length less than $\ell$. On the other hand, if $x$ is not on $C$, then we obtain a cycle of length $\ell+2$, with two linking edges, and the path in $X_{1}$ of length $j-2$.

When $X_{1}$ has valency at least 3 , we carry out the preceding modifications until we reach a cycle $C^{\prime}$ of odd length $\ell^{\prime}$ with two linking edges and a single edge in $X_{1}$. We then use Theorem 1.1(1) to replace the edge in $X_{1}$ with paths in $X_{1}$ of any odd length. As we proceed from $C$ to $C^{\prime}$, the length at each iteration either increases by 2 , remains the same or decreases. Thus, every odd length between $\ell$ and $\ell^{\prime}$ is covered if $\ell<\ell^{\prime}$. The result now follows.

When the valency of $X_{1}$ is 2 , then the initial path $Q$ is a subpath of a cycle generated by $s \in S_{1}$. We then perform the same kind of alteration extending the length of the path intersecting $X_{2}$ until we reach an odd length cycle with two linking edges using $m$ vertices of $X_{2}$. This completes the proof for two linking edges. 
The essential fact we use for the induction is the following.

Fact 1. Let $C^{\prime}$ be a cycle of odd length $\ell$ using $2 t \geq 4$ linking edges. The cycle $C^{\prime}$ intersects $X_{1}$ in $t$ vertex-disjoint paths $P_{1}, P_{2}, \ldots, P_{t}$, and intersects $X_{2}$ in $t$ vertex-disjoint paths $Q_{1}, Q_{2}, \ldots, Q_{t}$, where a path may be just a single vertex. If there is an edge between two vertices in distinct paths $P_{a}$ and $P_{b}$, or in distinct paths $Q_{c}$ and $Q_{d}$, then there is a cycle of odd length less than $\ell$ and using strictly fewer linking edges than $2 t$.

To see that this fact holds, suppose the vertices of the chord are $x$ and $y$. When we look at the two cycles which have only the edge $x y$ in common, we must use distinct linking edges in each of the paths containing $x$ and $y$, respectively, so that both cycles have fewer linking edges. One of the two cycles must have odd length and it is less than $\ell$. This establishes the fact.

Two linking edges is the induction basis and we let $C$ be a cycle of length $\ell$ with $2 t>2$ linking edges. Let $Q_{1}, Q_{2}, \ldots, Q_{t}$ be the paths forming the intersection of $C$ and $X_{2}$. Without loss of generality let $Q_{1}$ and $Q_{2}$ be the two paths that are closest together in $X_{2}$. Let the path of $X_{2}$ defining their distance apart be $w_{1} w_{2} \cdots w_{a}, w_{1} \in Q_{1}$ and $w_{a} \in Q_{2}$.

If $a=2$, that is, the edge $w_{1} w_{2}$ joins a vertex of $Q_{1}$ with a vertex of $Q_{2}$, then Fact 1 tells us there is a cycle $C^{\prime}$ of odd length $\ell^{\prime}<\ell$ with fewer linking edges. We then conclude there are cycles of all odd lengths from $\ell^{\prime}$ through $2 m-1$ by induction. So the conclusion holds for $C$.

When $a>2$, there are two subcases. First, if $w_{1}$ is an end vertex of $Q_{1}$, then let $s$ generate the edge from $w_{1}$ to $w_{2}$ and $s_{1} \in S_{2}$ generate a linking edges incident with $w_{1}$. Now let $x=w_{2} s_{1}$. If $x$ is not on $C$, then the $\left(w_{1}, w_{2}, x, w_{1} s_{1}\right)$-bypass produces a cycle of length $\ell+2$ with two paths in the intersection with $X_{2}$ closer together. If $x$ is on $C$, then we get an odd length cycle of length at most $\ell$ with fewer linking edges. We then use induction to get the result.

The second subcase is that $w_{1}$ is an internal vertex of $Q_{1}$. Let $w_{1} w_{2}$ be generated by $s \in S_{1}$ as we move from $w_{1}$ to $w_{2}$. Then one of the two edges on $Q_{1}$ incident with $w_{1}$ is generated by an $s^{\prime} \notin\left\{s, s^{-1}\right\}$. Let $v=w_{1} s^{\prime}$ and $x=v s$. If $x$ is not on $C$, then the $\left(w_{1}, w_{2}, x, v\right)$-bypass again gives a cycle of length $\ell+2$ with a shorter path joining two paths of its intersection with $X_{2}$. If $x$ is on $C$, then $x$ must be on $Q_{1}$ because we have assumed the distance between distinct paths in $X_{2}$ is at least 2. Let $Q^{\prime}$ be the subpath of $Q_{1}$ joining $x$ and $w_{1}$. If $Q^{\prime}$ has even length, then replacing $Q^{\prime}$ by the 2-path $x w_{2} w_{1}$ gives a new cycle of odd length whose intersection with $X_{2}$ has two paths that are closer together. If $Q^{\prime}$ has odd length, then $Q^{\prime}$ plus the same 2-path gives an odd cycle of length less than $\ell$ with no linking edges.

We continue until we either reach an odd length cycle with a chord between two paths in the intersection of the cycle with $X_{2}$, or at some 


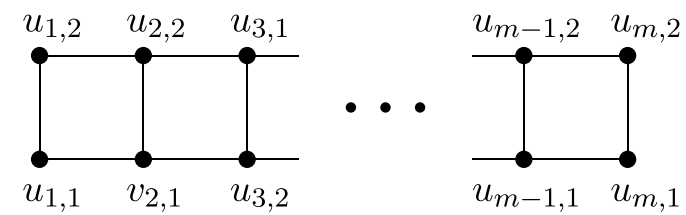

Figure 2.

iteration we get an odd length cycle with fewer linking edges. Both outcomes give us an odd length cycle with fewer linking edges. Because of the way the lengths change at each iteration, when we apply the induction to the cycle $C^{*}$ with fewer linking edges, all lengths between $\ell$ and the length of $C^{*}$ have been filled in if the length of $C^{*}$ happens to be larger than $\ell$.

This completes the argument for $X_{1}$ being connected.

\subsection{Disconnected case}

In this subsection we consider the case that $X_{1}$ is disconnected. We contract each component to a single vertex, remove all loops and reduce the multiplicity of any multiple edges to 1 . The components of $X_{1}$ are Cayley graphs on a subgroup $K$ of $H$ and the contracted graph is a Cayley graph $\bar{X}$ on the generalized dihedral group $D_{\bar{H}}$, where $\bar{H}$ is the quotient group $H / K$. This graph $\bar{X}$ has a Hamilton path by Lemma 2.2. When we return to $X$, the Hamilton path in $\bar{X}$ yields a spanning subgraph of $X$ isomorphic to the cartesian product $P \square X^{\prime}$, where $X^{\prime}$ is a component of $X_{1}$ and has order $r$ and $P$ is the path with $2 m / r$ vertices.

When $X_{1}$ has valency $1, X^{\prime}$ is a single edge, and $2 m / r=m$. The spanning subgraph is shown in Figure 2. When $X_{1}$ has valency $2, X^{\prime}$ is a cycle of length $r$. When $X_{1}$ has valency 3 or more, we have a spanning subgraph isomorphic to $P \square X^{\prime}$. We choose a spanning cycle of $X^{\prime}$ in the first copy of $X^{\prime}$ and obtain copies of this spanning cycle in successive components by multiplying on the right using the element of $S_{2}$ that generates the perfect matching joining the two components (Lemma 2.1). In this way we obtain a spanning subgraph of $X$ isomorphic to $P \square C^{\prime}$, where $C^{\prime}$ is a cycle of length $r$ when the valency of $X_{1}$ is 2 or more. We denote this spanning subgraph by $Y$ for the rest of the paper.

We coordinatize the vertices of $Y$ using $u_{i, j}$ for $i=1,2, \ldots, 2 m / r$ and $j=1,2, \ldots, r$. Edges of the form $u_{i, j} u_{i, k}$ are called vertical edges, edges of the form $u_{i, j} u_{i^{\prime}, j}$ are called horizontal edges, and all other edges are called diagonal edges. All subscript arithmetic in the second coordinate is done modulo $r$. 
Starting with the 4-cycle $u_{1,1} u_{2,1} u_{2,2} u_{2,1} u_{1,1}$, it is easy to see that we can find cycles of all even lengths using the edge $u_{1,1} u_{1,2}$ or the edge $u_{1,1} u_{2,1}$. The edge $u_{1,1} u_{2,1}$ is a linking edge and from Lemma 2.2, we see that we may choose this edge to be generated by any element of $S_{2}$. Similarly, because $X^{\prime}$ is a Cayley graph on an abelian group, we may choose $u_{1,1} u_{1,2}$ to be generated by any $s \in S_{1}$. Because $X$ is vertex-transitive, we get that every edge belongs to cycles of all even lengths. Therefore, $X$ is even edge-pancyclic.

The preceding argument takes care of the case that $X$ is bipartite. The rest of the proof deals with odd length cycles and the length attests to the fact that they are troublesome. We now give several useful facts.

Fact 2. If $u_{i, j} u_{i^{\prime} j^{\prime}}$ is a non-vertical edge in $X$, then $i$ and $i^{\prime}$ have different parity. This follows because column $k$ and column $k^{\prime}$ are components in the same $X_{a}, a=1,2$, if and only if $k$ and $k^{\prime}$ have the same parity.

Fact 3. If $C$ is an odd length cycle in $X$, then $C$ has an odd number of vertical edges. This follows because Fact 2 implies that the number of non-vertical edges in $C$ must be even.

Fact 4. Let $C$ be a cycle of length $\gamma_{o}(X)$. If $e$ is a vertical edge followed by a non-vertical edge $e^{\prime}$ on $C$ forming a 2-path from vertex $x$ to vertex $y$, then there is a 2-path from $x$ to $y$ formed by a non-vertical edge followed by a vertical edge $e^{\prime \prime}$, where $e$ is generated from $x$ by $s$ and $e^{\prime \prime}$ is generated into $y$ by $s^{-1}$. This follows because if $y=x s s_{1}$, then $y=x s_{1} s^{-1}$, where $s_{1} \in S_{2}$.

Note that replacing the initial 2-path by the latter 2-path gives us another cycle of length $\gamma_{o}(X)$. We refer to this as moving the vertical edge along $C$.

We now proceed by increasing valency starting with $X_{1}$ having valency 1. Referring to Figure 2, we see that the spanning subgraph $Y$ determines an implicit bipartition of $X$. Thus, the reason that $X$ is not bipartite is that there is a linking edge $e$ of $X$ violating this implicit bipartition. Because of Fact 2, this edge has the form $u_{i, 1} u_{i^{\prime}, 2}$, where $i<i^{\prime}$ and $i^{\prime}-i$ is odd (this is valid because $u_{i, 1} u_{i^{\prime}, 2}$ belongs to $X$ if and only $u_{i, 2} u_{i^{\prime}, 1}$ belongs to $X$ ).

Let $C^{\prime}$ be the odd length cycle containing $u_{i, 2} u_{i, 1} u_{i^{\prime}, 2}$ and the path in the top row from $u_{i, 2}$ to $u_{i^{\prime} 2}$. We can then use successive horizontal 3-bypasses to the left starting with the vertical edge $u_{i, 1} u_{i, 2}$ until we have used all the vertices to the left of column $i$. Call this last cycle $C^{\prime \prime}$.

The subpath of $C^{\prime \prime}$ from $u_{i, 2}$ to $u_{i^{\prime}, 2}$ along the top row has odd length. If this odd length is $\ell>1$, then choose the matching with $(\ell-1) / 2$ edges from this subpath such that the matching edges use neither vertex $u_{i, 2}$ nor $u_{i^{\prime}, 2}$. We can then use a 3-bypass vertically on each edge of the matching edges until we reach a cycle $C^{*}$ using all the vertices of the first $i^{\prime}$ columns except $u_{i^{\prime}, 1}$. So $C^{*}$ has length $2 i^{\prime}-1$ and we are done when $i^{\prime}=m$. 
When $i^{\prime}<m, C^{*}$ contains the 4 -path $u_{i^{\prime}-2,2} u_{i^{\prime}-2,1} u_{i^{\prime}-1,1} u_{i^{\prime}-1,2} u_{i^{\prime}, 2}$. We replace it by the 6 -path $u_{i^{\prime}-2,2} u_{i^{\prime}-1,2} u_{i^{\prime}-1,1} u_{i^{\prime}, 1} u_{i^{\prime}+1,1} u_{i^{\prime}+1,2} u_{i^{\prime}, 2}$. We now have a vertical edge on the right from which we can construct 3 -bypasses to the right to reach a cycle of length $2 m-1$. This produces cycles of all lengths from $\left|V\left(C^{\prime}\right)\right|$ through $2 m-1$.

The preceding does not work if the subpath of $C^{\prime \prime}$ has length 1 , but this is not a problem as we can use Lemma 2.2 to make sure the corresponding linking edge is between columns 1 and 2 in forming $Y$. Then the 3 -cycle $C^{\prime}$ is $u_{1,1} u_{2,1} u_{2,2} u_{1,1}$. We now carry out successive bypasses to the right to achieve cycles of all odd lengths from 3 through $2 m-1$.

We have shown that we can fill in all odd lengths from the length of any cycle $C$ coming from a single edge violating the implicit bipartition through $2 m-1$. The violating edge yielding the worst case is $u_{1,1} u_{m, 2}$ which gives a cycle of length $m+1$. Thus, it suffices to fill in the odd lengths from $\gamma_{o}(X)$ through $m-1$.

Now let $C$ be a cycle of length $\gamma_{o}$ in $X$. The cycle $C$ may be drawn on the vertices of $Y$ and it may or may not use some edges of $Y$. We want to examine the structure of $C$.

If both vertices of a column in $Y$ belong to $C$, then the edge joining them must also be in $C$ or else that edge would be a chord of $C$, and cycles of odd length $\gamma_{o}$ have no chords. We claim that $C$ has exactly one column edge of $Y$ belonging to it. We know it has at least one by Fact 3. If $C$ has at least two column edges, then using Fact 4 we get a cycle of length $\gamma_{o}(X)$ with two vertical edges joined by a non-vertical edge. But this implies that we have a cycle of length $\gamma_{o}(X)$ with a chord because there is a non-vertical edge joining the other ends of the vertical edges. This is impossible.

Note that for all columns, other than the column containing the vertical edge, at most one vertex of the column belongs to $C$ because it has no chords. Given an edge $x z$ between distinct columns, we call the 3-bypass $x x^{\prime} z^{\prime} z$, where $x^{\prime}, z^{\prime}$ are the other vertices in the same respective columns, the vertical bypass on the edge $x z$. Our general procedure is to choose a matching from the edges of $C$ that are not column edges and perform successive vertical bypasses on each edge of the matching, thereby, adding two to the length at each iteration.

We may assume that $\gamma_{o}(X)>3$ because we know a 3 -cycle must contain a vertical edge and earlier we showed how to go from a 3-cycle to pick up all odd lengths through $2 m-1$. Let $i_{1}<i_{2}<\cdots<i_{t}$ be the column indices used by vertices of $C$. We know $t$ is even as $C$ has one column edge. Moreover, there is a cycle induced on the set of indices by $C$. There are two perfect matchings of this induced cycle because $t$ is even and $t \geq 4$. Choose a 
perfect matching so that the vertices in columns $i_{t}$ and $i_{t-1}$ are on different matching edges $e$ and $e^{\prime}$, respectively.

If $i_{t-1} \leq m / 2$, move the column edge so that it is in column $i_{t}$ and do a vertical bypass on the edge $e^{\prime}$. We then have a cycle of length $\gamma_{o}(X)+2$ with vertical edges in columns $i_{t-1}$ and $i_{t}$ and no vertex of the cycle in any other column to the right of column $i_{t-1}$. Then we use horizontal bypasses to the right from these two columns to reach a cycle of odd length bigger than $m$. On the other hand, if $i_{t-1}>m / 2$, then move the column edge to the column containing the end of $e$ not in column $i_{t}$ and perform vertical bypasses on all edges different from $e$. We now can do horizontal bypasses to the left from all columns $i_{t-1}$ and smaller. The resulting cycle has odd length bigger than $m$ and this completes the proof for $X_{1}$ having valency 1 .

We now move to the case that the valency of $X_{1}$ is at least 2 . Recall that the spanning subgraph $Y$ is isomorphic to $P \square C$, where $C$ is a cycle of length $r$ and $P$ is a path of order $2 m / r$. The rest of the proof deals only with odd length cycles because we did the bipartite case earlier. We find the convenient odd length cycles first.

If $X_{1}$ is not bipartite, then choose a vertical cycle of length $r$ spanning column 1 when $r$ is odd, or of length $r-1$ when $r$ is even. It is now easy to see how we can use horizontal bypasses to the right and the fact that the columns contain a spanning cycle to obtain cycles of all odd lengths from $r-1$ or $r$ through $2 m-1$

If $X_{1}$ is bipartite, then $Y$ gives us an implicit bipartiton of $X$. There must be an edge $u_{i, j} u_{i^{\prime}, k}, i<i^{\prime}$, violating the implicit bipartition because $X$ is not bipartite. Without loss of generality we may assume $j \leq k$. The cycle

$$
C^{\prime}=u_{i, j} u_{i+1, j} \cdots u_{i^{\prime}, j} u_{i^{\prime}, j+1} \cdots u_{i^{\prime}, k} u_{i, j}
$$

has odd length and the maximum this length may be is $r / 2+2 m / r$. There are several subcases to check, but it is routine to verify that there are cycles of all odd lengths from the length of $C^{\prime}$ through $2 m-1$.

To complete the proof we need to show that we can fill in all odd lengths from $\gamma_{o}(X)$ through the larger of the odd parts of $m$ and $r / 2+2 m / r$. Throughout the rest of the proof, $C$ denotes a cycle of length $\gamma_{o}(X)$.

Fact 5. We may assume that all the vertical edges of $C$ form a subpath of $C$ lying in a single column different from column 1 or column $2 \mathrm{~m} / r$. We use Fact 4 to transform the cycles of length $\gamma_{o}(X)$ until the vertical edges form a path. To see that we may assume this path lies in a column different from 1 or $2 \mathrm{~m} / \mathrm{r}$, first observe that if $C$ has only vertices in columns 1 and $2 \mathrm{~m} / \mathrm{r}$, then it is easy to find cycles of all appropriate odd lengths via the following argument. From Fact 3 we know there is a vertical edge. We may use this 
vertical edge to give horizontal bypasses to the left or right to the column next to the other boundary column. We then use one of the horizontal edges formed earlier and vertical bypasses to hit every row not used there. This gives us a cycle of odd length bigger than $r+2 m / r$ and we are done. So we may assume that $C$ uses a vertex in another column and we use Fact 4 to move the vertical edges to this column.

We now start the general argument. Let $C$ be a cycle of length $\gamma_{o}(X)$ with the maximum number of vertical edges such that they form a subpath $P$ lying in a single column different than column 1 and column $2 \mathrm{~m} / \mathrm{r}$. We use this to get three further useful facts.

Fact 6. We may assume that $\gamma_{o}(X) \geq 5$. This follows because if $\gamma_{o}(X)=$ 3 , then a 3-cycle has one or three vertical edges by Fact 3 . There are a couple of cases to check, but it is easy to use horizontal and vertical bypasses to find cycles of all odd lengths from 3 through the maximum of the odd part of $r / 2+2 m / r$.

Fact 7. Let $U$ be the vertices of $C$ not lying on $P$. If $u_{i, j} \in U-\left\{w_{1}, w_{2}\right\}$, where $w_{1}$ and $w_{2}$ are the end vertices not on $P$ of the two non-vertical edges of $C$ adjacent with $P$, and $u_{i^{\prime}, j^{\prime}}$ is a vertex of $C$, then whenever $i$ and $i^{\prime}$ have different parity, there is no 2-path $u_{i, j} v u_{i^{\prime}, j^{\prime}}$ joining them in $X$ with $v \notin V(C)$. Continuing, if $i=i^{\prime}$, then they have no common neighbor in column $i$. Similarly, if $u_{i, j}$ and $u_{i+2, j}$ belong to $C$, then we may assume that the 2-path $u_{i, j} u_{i, j+1} u_{i, j+2}$ belongs to $C$.

To see that Fact 7 holds, let $u_{i, j} \in U-\left\{w_{1}, w_{2}\right\}$ and suppose there is a 2-path $u_{i, j} v u_{i^{\prime}, j^{\prime}}$ in $X$ with $v$ not on $C$ and $u_{i^{\prime}, j^{\prime}}$ on $C$. There is no edge joining $u_{i, j}$ and $u_{i^{\prime}, j^{\prime}}$ by Fact 6 . Thus, the vertices would be distance 2 apart in $X$ and so the same distance apart on $C$. However, such a 2-path must contain a vertical edge by Fact 2 which contradicts the vertical edges of $C$ forming a path. We also see why $w_{1}$ and $w_{2}$ are possible exceptions.

If $i=i^{\prime}$, then a common neighbor in column $i$ would give a 2-path of vertical edges joining $u_{i, j}$ and $u_{i, j^{\prime}}$ again contradicting the choice of $C$. Finally, if $u_{i, j}$ and $u_{i+2, j}$ belong to $C$, then the two vertices must be distance 2 apart on $C$ and the 2-path currently joining them must consist of two nonvertical edges by Fact 2 . Replace it by the 2 -path $u_{i, j} u_{i, j+1} u_{i, j+2}$ and we still have a cycle of length $\gamma_{o}(X)$.

Fact 8. If $u_{i, j}$ is a vertex of $C$ and there is an edge $e$ of $X$ joining $u_{i, j}$ and $u_{i^{\prime}, j^{\prime}}$ such that $e$ does not belong to $C$, then $u_{i^{\prime}, j^{\prime}}$ does not belong to $C$. To see this fact, note that $u_{i^{\prime}, j^{\prime}}$ cannot belong to $C$ as $e$ would then be a chord.

In the special case that there are exactly two non-vertical edges in $C$, then $C$ consists of a path in column $k$ with one vertex in another column. It is easy to see how to get cycles of all odd lengths from $\gamma_{o}(X)$ through at 
least $r+2 m / r$ because either column $k-1$ or column $k+1$ has no vertices of $C, r \geq 4$ and the lone vertex blocks only one row and one column. So we may assume there are at least four non-vertical edges.

We perform a sequence of 3-bypasses on $C$ starting with the subpath $P$ of vertical edges in $C$ in column $k \notin\{1,2 m / r\}$. Let $u_{k, a}$ and $u_{k, b}$ be the end vertices of $P$ in column $k$. If the non-vertical edge incident with $u_{k, a}$ is not $u_{k-1, a} u_{k, a}$, then do a horizontal 3-bypass to the left on the vertical edge of $P$ incident with $u_{k, a}$. Follow with a horizontal 3-bypass to the right on the next edge of $P$. Continue in this way alternating horizontal bypasses to the left and right. When you reach the last edge of $P$-incident with $u_{k, b}$-do a horizontal 3-bypass to the left unless the edge $u_{k-1, b} u_{k, b}$ already belongs to $C$ in which case we omit the last horizontal 3-bypass. On the other hand, if the non-vertical edge incident with $u_{k, a}$ is $u_{k-1, a} u_{k, a}$, then we reverse the role of left and right horizontal bypasses. Let $C^{*}$ denote the cycle we have after carrying out the bypasses on the edges of $P$.

We now carry out a sequence of 3-bypasses on the non-vertical edges of $C$ working around the cycle in a cyclic fashion starting with the non-vertical edge $u_{k, a} u_{i, j}$ incident with $u_{k, a}$. We do not carry out a bypass on this edge. We move to the next edge $u_{i, j} u_{i^{\prime}, j^{\prime}}$ on $C$. If $u_{i, j+1}$ is on $C^{*}$, then we take no bypass on the edge $u_{i, j} u_{i^{\prime}, j^{\prime}}$. On the other hand, if $u_{i, j+1}$ is not on $C^{*}$, let the edge $u_{i, j} u_{i, j+1}$ be generated by $s \in S_{1}$. We then take the $(s, t, s)$-bypass on the edge $u_{i, j} u_{i^{\prime}, j^{\prime}}$, where $t$ generates the edge $u_{i, j} u_{i^{\prime}, j^{\prime}}$.

Let $u_{i^{\prime}, j^{\prime}} u_{i^{\prime \prime}, j^{\prime \prime}}$ be the next edge of $C$. If the edge $u_{i^{\prime}, j^{\prime}+1} u_{i^{\prime}, j^{\prime}}$ is not an edge of the preceding bypass, then take the 3 -bypass on the edge $u_{i^{\prime}, j^{\prime}} u_{i^{\prime \prime}, j^{\prime \prime}}$ that starts with $u_{i^{\prime}, j^{\prime}} u_{i^{\prime}, j^{\prime}+1}$. But if the edge $u_{i^{\prime}, j^{\prime}+1} u_{i^{\prime}, j^{\prime}}$ is an edge of the preceding bypass, then take the 3-bypass on the edge $u_{i^{\prime}, j^{\prime}} u_{i^{\prime \prime}, j^{\prime \prime}}$ that starts with $u_{i^{\prime}, j^{\prime}} u_{i^{\prime}, j^{\prime}-1}$.

We continue this way until reaching the last non-vertical edge as we work along $C$, and we do not perform a 3-bypass on this last edge which is incident with $u_{k, b}$. We want the final subgraph to be a cycle and we may need to make a small adjustment near the end of the procedure. In order to do so, we must deal with the following. If $C$ has a vertex in row $j$, a vertex in row $j+\alpha>j+1$, but none in the rows in between, then we let $\operatorname{gap}(j)=\{j+1, j+2, \ldots, j+\alpha-1\}$ (recall that we work modulo $r$ in the second coordinate). We continue with the verification that we finish with a cycle (after a possible adjustment).

The initial horizontal bypasses on the vertical edges of $P$ bring in new vertices none of which lie on $C$ by Fact 8 . All remaining new vertices result from bypasses on non-vertical edges. Two bypasses in the same column cannot require the same vertex by Fact 7 as the vertical edges would have a common neighbor. No vertical edge that is part of a bypass on a non-vertical 
edge requires a vertex that arose from the initial horizontal bypasses by Fact 7 because we would then have two vertices of $C$ in adjacent columns at distance 2 .

Note that we avoided the duplication problem at the beginning when $u_{i, j+1}$ lies on $C^{*}$ as we did not perform a 3 -bypass on the edge $u_{i, j} u_{i^{\prime}, j^{\prime}}$ in that situation. The bypasses at the end of the procedure may require adjustment. We do no bypass on the non-vertical edge $u_{k^{\prime}, b^{\prime}} u_{k, b}$ incident with $u_{k, b}$. However, a new vertical edge may now be present at $u_{k^{\prime}, b^{\prime}}$ because of a bypass on the preceding edge $u_{i, j} u_{k^{\prime}, b^{\prime}}$. If the new vertical edge does not encounter a vertex added earlier, then we leave the bypass intact. On the other hand, if the new vertical edge encounters a vertex $v$ that arose earlier, then $v$ had to come on a horizontal bypass for $P$ (note that this means $k^{\prime}=k-1$ or $\left.k^{\prime}=k+1\right)$. But this means there already is a horizontal edge in the row containing $v$ so that we are free to make an adjustment to the bypass on the edge $u_{i, j} u_{k^{\prime}, b^{\prime}}$.

If $\operatorname{gap}\left(b^{\prime}\right)$ does not exist, that is, there is a vertex of $C$ in row $b^{\prime}+1$, then we do not perform a bypass on $u_{i, j} u_{k^{\prime}, b^{\prime}}$ and the problematic vertical edge does not arise. Assume then that gap $\left(b^{\prime}\right)$ does exist which implies that the vertex $u_{k^{\prime}, b^{\prime}+1}$ is not on $C$. Let $B_{1}$ denote the bypass on $u_{i, j} u_{k^{\prime}, b^{\prime}}$ which finishes with the edge $u_{k^{\prime}, b^{\prime}+1} u_{k^{\prime}, b^{\prime}}$. If the other end of $B_{1}$ is $u_{i, j} u_{i, j^{\prime}}$ and is not used in the bypass $B_{2}$ on the preceding edge adjacent to $u_{i, j} u_{k^{\prime}, b^{\prime}}$, then substitute $B_{1}$ for the bypass containing the problematic vertical edge.

The preceding substitution cannot be done if the edge $u_{i, j} u_{i, j^{\prime}}$ is used in $B_{2}$. Then if $\operatorname{gap}(j)$ does not exist, remove the edge $u_{i, j} u_{i, j^{\prime}}$ from $B_{1} \cup B_{2}$ and we have a path of length 4 replacing a subpath of length 2 in $C$ such that the edge $u_{k^{\prime}, b^{\prime}} u_{k^{\prime}, b^{\prime}+1}$ is in the path. Finally, if $\operatorname{gap}(j)$ does exist, then we compare $|\operatorname{gap}(j)|$ and $\operatorname{gap}\left(b^{\prime}\right) \mid$ as one of them will be 'sacrificed.' If $\left|\operatorname{gap}\left(b^{\prime}\right)\right|$ is smaller, then perform no bypass on the edge $u_{i, j} u_{k^{\prime}, b^{\prime}}$. If $\left|\operatorname{gap}\left(b^{\prime}\right)\right|$ is equal or larger, then carry out the 4-path substitution.

Therefore, we obtain a cycle $C^{\prime}$ after carrying out the sequence of alterations above. We have introduced many vertical edges that may be used for horizontal bypasses to fill column gaps. Normally (exceptions are discussed next) $C^{\prime}$ has vertical edges in columns $k-1, k+1$ and any column $i, i \notin\{k-1, k, k+1\}$, which contained a vertex of $C$. Also, no vertices are introduced to columns not containing a vertex of $C$ other than $k-1$ and $k+1$. So horizontal bypasses to the left fill in all column gaps to the left of column $k-1$ and bypasses to the right take care of gaps to the right of column $k+1$. This gives us a cycle of odd length at least $4 \mathrm{~m} / \mathrm{r}$.

One exceptional situation is when $P$ has length 1 . There are a few subcases to be checked which we leave to the reader but here are a few hints. If a new vertical edge has been added to column $k$, then the edge of $P$ may be 
used for bypasses to the left and the new edge for bypasses to the right and the column gaps may be filled. If no new edge is added to column $k$, then column $k$ contains only two vertices of $C$. This allows us to have room to do a vertical bypass on a horizontal edge adjacent to the edge of $P$ in $C^{\prime}$. That then gives us a new vertical edge in column $k$ enabling us to now carry out the column gap filling.

The second exceptional situation comes from doing a 4-path substitution described above. This is column $i$ in the notation above. If another vertex of column $i$ lies in $C$, then we already have a vertical edge in column $i$ and there is no problem. Otherwise, $C^{\prime}$ has just one vertex $u_{i, j^{\prime}}$ in column $i$ and it might block horizontal bypasses we need. Let's build a bypass on the edge $u_{i, j^{\prime}} u_{k^{\prime}, b^{\prime}+1}$. One of $u_{i, j^{\prime}+1}$ and $u_{i, j^{\prime}-1}$ is not the original $u_{i, j}$ that we eliminated and we assume it is $u_{i, j^{\prime}-1}$. Let $t$ generate the edge from $u_{i, j^{\prime}}$ to $u_{k^{\prime}, b^{\prime}}$. Now consider the potential bypass starting $u_{i, j^{\prime}} u_{i, j^{\prime}-1}$ and followed by the edge generated by $t$ taking us to vertex $x$ in column $k^{\prime}$.

If $x$ is not on $C^{\prime}$, then we have a bypass on the edge $u_{i, j^{\prime}} u_{k^{\prime}, b^{\prime}+1}$ giving us a vertical edge in column $i$ so that we may complete the horizontal bypasses as required. If $x$ is on $C^{\prime}$, then it is on a vertical edge not involving the vertices $u_{k^{\prime}, b^{\prime}}$ or $u_{k^{\prime}, b^{\prime}+1}$. Thus, there is a horizontal bypass to column $i$ on this vertical edge again giving us a vertical edge in column $i$.

Hence, we reach a cycle with odd length at least $4 \mathrm{~m} / r$. Therefore, if $r \leq 4 m / r$, the final cycle has length at least $r$ and at least $4 m / r=2 m / r+$ $2 m / r \geq r / 2+2 m / r$.

The preceding completes the argument when $r \leq 4 m / r$. So we now assume that $r>4 m / r$ and we look at gaps involving rows rather than columns. The argument is more complicated now. We need some facts again.

Fact 9. Let $u_{i, j}$ be a vertex of $C$ not lying on $P$. Any one of the vertices $u_{i-1, j}$ and $u_{i+1, j}$ belongs to $C^{\prime}$ if and only if the corresponding edge $u_{i-1, j} u_{i, j}$ or $u_{i, j} u_{i+1, j}$, respectively, belongs to $C$. This follows from Facts 6,7 and 8 .

Fact 10. If two edges $u_{i, j^{\prime}} u_{i, j}$ and $u_{i+1, j^{\prime \prime}} u_{i+1, j}$ both are added as a result of bypasses, where $u_{i, j^{\prime}}$ and $u_{i+1, j^{\prime \prime}}$ are vertices of $C$, then we may assume that $u_{i, j^{\prime}} u_{i+1, j^{\prime \prime}}$ is an edge of $C$. Let's prove this fact.

We can see that the distance between $u_{i, j^{\prime}}$ and $u_{i+1, j^{\prime \prime}}$ is at most 3 in $X$. If the distance is 3 , then that must be their distance apart on $C$ and the path joining them on $C$ can have at most one vertical edge because both vertices have admitted bypasses. We can then replace the 3-path joining them by the 3-path $u_{i, j^{\prime}} u_{i, j} u_{i+1, j} u_{i+1, j^{\prime \prime}}$ which has two vertical edges contradicting the choice of $C$.

If the distance between them in $X$ is 2, then a vertical edge is involved but this is impossible. Hence, the distance between them is 1 and the edge must then be part of $C$. 
The strategy now is clear. For any row bounding a gap for $C^{\prime}$, we want to find a horizontal edge in that row. We may then fill the gap by successive vertical bypasses on the horizontal edge. Here is the pillar for that strategy. Suppose $u_{i, j}$ is a vertex of $C$ and we have added the edges $u_{i, j} u_{i, j+1}$ and $u_{i, j} u_{i, j^{\prime}}$ in building $C^{\prime}$. Further, suppose that $u_{i+1, j}, u_{i+1, j+1}$ and $u_{i+1, j^{\prime}}$ do not lie on $C^{\prime}$. Then if both $\operatorname{gap}(j+1)$ and $\operatorname{gap}\left(j^{\prime}\right)$ exist, we may do the following. The horizontal $\left(u_{i, j}, u_{i+1, j}, u_{i+1, j+1}, u_{i, j+1}\right)$-bypass on the edge $u_{i, j} u_{i, j+1}$ yields a horizontal edge in row $j+1$. Now replace the 2 -path $u_{i+1, j} u_{i, j} u_{i, j^{\prime}}$ with the 2-path $u_{i+1, j} u_{i+1, j^{\prime}} u_{i, j^{\prime}}$ to also attain a horizontal edge in row $j^{\prime}$. Of course, it is easy to get a horizontal edge in only one of the rows if that is all that is required.

Any row containing a vertex of $P$ acquires a horizontal edge at the beginning so that we are interested only in gap rows arising from vertical bypass edges. So for a given $u_{i, j}$ incident with two bypass edges $u_{i, j} u_{i, j+1}$ and $u_{i, j} u_{i, j^{\prime}}$, we consider $j^{\prime}=j-1$ and $j^{\prime} \neq j-1$ separately. From Fact 9 we know that $u_{i+1, j}$ is a vertex of $C^{\prime}$ if and only if the edge $u_{i, j} u_{i+1, j}$ is in $C$. So we first examine the subcase that $u_{i+1, j}$ is in $C^{\prime}$ and assume we traverse $C$ in the direction from $u_{i, j}$ to $u_{i+1, j}$.

When $j^{\prime} \neq j-1$, the 3 -path $u_{i, j} u_{i, j+1} u_{i+1, j+1} u_{i+1, j}$ is the bypass on $u_{i, j} u_{i+1, j}$. This already gives a horizontal edge in row $j+1$. If we need a horizontal edge in row $j^{\prime}$, then because $u_{i, j-1}$ is not on $C^{\prime}$ by Facts 7 and 8 , we may replace the 5 -path

$$
u_{i, j^{\prime}} u_{i, j} u_{i, j+1} u_{i+1, j+1} u_{i+1, j} u_{i+1, j-1}
$$

with the 7-path

$$
u_{i, j^{\prime}} u_{i+1, j^{\prime}} u_{i+1, j} u_{i+1, j+1} u_{i, j+1} u_{i, j} u_{i, j-1} u_{i+1, j-1}
$$

and obtain horizontal edges in both row $j+1$ and row $j^{\prime}$.

When $j^{\prime}=j-1$, then one possibility is that we have the same 3-path as in the preceding paragraph. We already have a horizontal edge in row $j+1$ and $\operatorname{gap}\left(j^{\prime}\right)$ does not exist so we are done.

The other possibility is that we have the 5-path

$$
u_{i, j+1} u_{i, j} u_{i, j-1} u_{i+1, j-1} u_{i+1, j} u_{i+1, j+1}
$$

because of the way we construct the bypasses. So in this case we make no further changes unless we need a horizontal edge in row $j+1$. If either edge that is adjacent to $u_{i, j} u_{i+1, j}$ is horizontal, then we have a horizontal edge in row $j+1$ because of the way the bypasses work. If neither of the adjacent edges is horizontal, then $u_{i+2, j}$ is not on $C^{\prime}$ by Fact 9 . The vertex $u_{i+2, j+1}$ 
also is not on $C^{\prime}$ because of Fact 10. We can then do a horizontal bypass to the right on the vertical edge $u_{i+1, j} u_{i+1, j+1}$ to obtain a horizontal edge in row $j+1$. We may do the same to the left if column $i+2$ does not exist.

We now consider the other case that $u_{i+1, j}$ is not on $C^{\prime}$. The edge $u_{i, j+1} u_{i+1, j+1}$ is not part of the bypass starting with $u_{i, j} u_{i, j+1}$ because this would force the edge $u_{i, j} u_{i+1, j}$ to be on $C$. Thus, if $u_{i+1, j+1}$ is on $C^{\prime}$, it arises from a bypass edge. Fact 10 then tells us that it must be from an edge of $C$ incident with $u_{i, j}$. But this edge is not $u_{i, j} u_{i+1, j}$ so that the bypass cannot go through $u_{i+1, j+1}$. So neither $u_{i+1, j}$ nor $u_{i+1, j+1}$ belong to $C^{\prime}$ and we are free to use a horizontal bypass to the right on $u_{i, j} u_{i, j+1}$. We now have a horizontal edge in row $j+1$.

Now $\operatorname{gap}\left(j^{\prime}\right)$ exists only when $j^{\prime} \neq j-1$. If the vertex $u_{i+1, j^{\prime}}$ is not on $C^{\prime}$, then we may use the earlier mentioned pillar for the strategy. On the other hand, if $u_{i+1, j^{\prime}}$ does lie on $C^{\prime}$, then Fact 10 tells us that we have the 4-path $u_{i, j^{\prime}} u_{i, j} u_{i, j+1} u_{i+1, j^{\prime}} u_{i+1, j^{\prime \prime}}$ after applying a bypass on the edge $u_{i, j} u_{i+1, j^{\prime \prime}}$. We may then replace that 4 -path by the 6 -path

$$
u_{i, j^{\prime}} u_{i+1, j^{\prime}} u_{i, j+1} u_{i+1, j+1} u_{i+1, j} u_{i, j} u_{i+1, j^{\prime \prime}}
$$

to get horizontal edges in both row $j+1$ and row $j^{\prime}$.

The last thing to examine is the issue of the adjustment we may have made for the edge returning to $P$ at vertex $u_{k, b}$. When we add vertices to a row to fill in a gap, we add two vertices to the row. Thus, even if we obtain no new vertices in the smaller of $\operatorname{gap}(c)$ and $\operatorname{gap}(j)$, we add at least $|\operatorname{gap}(c)|+|\operatorname{gap}(j)|$ vertices for the rows in these two gaps. This implies that the final cycle has at least length $r$. Because $r>4 m / r$, the length of the cycle is greater than $r$ and $r / 2+2 m / r$ as required. This completes the proof.

\section{Final comments}

The glaring omission in searching for an analogue of Theorem 1.1 for the family of Cayley graphs on generalized dihedral groups is the case that $X_{1}$ (and thus $X_{2}$ ) has no edges, that is, $S_{1}=\emptyset$. This is, of course, no surprise because we do not know whether or not all connected Cayley graphs on the dihedral group are hamiltonian. It is known that [3] all connected trivalent graphs on dihedral groups are hamiltonian. The identical proof for the aforementioned result works to prove the following so we omit the proof.

Theorem 4.1. If $X$ is a connected trivalent graph on a generalized dihedral group, then $X$ has a Hamilton cycle. 
The preceding theorem suggests that perhaps we can say something about pancyclicity for Cayley graphs on generalized dihedral groups when $S_{1}=\emptyset$ and $\left|S_{2}\right|=3$ because at least we know the longest cycle is there. The graphs are bipartite so that we may achieve only even length cycles.

The definition of $\operatorname{HTG}(m, n, \ell)$ still makes sense when $m=1$ as the honeycomb toroidal graph is then a Hamilton cycle of length $n$ with alternating chords of some odd length, but in this case $n>4$ must hold. We refer to these as degenerate honeycomb toroidal graphs, but they include important graphs. For example, the Heawood graph is $\operatorname{HTG}(1,14,5)$. The only reason we call these degenerate is because there is a natural embedding of honeycomb toroidal graphs on the torus and when $m=1$, there is the possibility that the hexagonal faces have a boundary walk that uses edges more than once.

The Heawood graph is an interesting example to consider. It is not hard to verify that it has girth 6 and cycles of all even lengths from 6 through 14 . So here is an example of a graph in our family of Cayley graphs not having cycles of length 4 . Because of this example, might it be possible that they miss at most cycles of length 4 ?

Proposition 4.2. The degenerate honeycomb toroidal graph $\operatorname{HTG}(1, n, \ell)$ has girth 4 if and only if one of the following holds: $\ell=3, \ell=n-3$, $\ell=n / 2$ and $n \equiv 2(\bmod 4)$, or $\ell=(n \pm 2) / 2$ and $n \equiv 0(\bmod 4)$.

Proof. Let $X=\operatorname{HTG}(1, n, \ell)$ and label the vertices $u_{0}, u_{1}, \ldots, u_{n-1}$. Recall from above that $n>4$ must hold. If $\ell=3$, then $u_{1} u_{2} u_{3} u_{4} u_{1}$ is a 4 -cycle. If $\ell=n-3$, then $u_{0} u_{1} u_{2} u_{3} u_{0}$ is a 4 -cycle. Similarly, when $n \equiv 2(\bmod 4)$ and $\ell=n / 2$, we find the 4 -cycle $u_{1} u_{1+n / 2} u_{n / 2} u_{0} u_{1}$ in $X$. Finally, when $n \equiv 0(\bmod 4)$, we have the 4 -cycle $u_{0} u_{1} u_{n / 2} u_{1+n / 2} u_{0}$, when $\ell=(n-2) / 2$, and the 4-cycle $u_{1} u_{2} u_{(n+2) / 2} u_{(n+4) / 2} u_{1}$ when $\ell=(n+2) / 2$.

We have seen that we have 4 -cycles when any of the four conditions hold. We need to show that this is the only way 4-cycles may arise. A 4-cycle must use at least one chord because $n>4$. If exactly one chord is used, then the three edges from the outside Hamilton cycle must form a subpath of length 3 in the 4 -cycle. Hence, $\ell$ is either 3 or $n-3$.

Now suppose a 4-cycle uses more than one chord. Chords are not adjacent so that there must be two chords, and these two chords must alternate with edges from the Hamilton cycle. So the 4-cycles have either the form $u_{i} u_{i+1} u_{j+1} u_{j} u_{i}$ or $u_{i} u_{i+1} u_{j} u_{j+1} u_{i}$.

If the 4-cycle has the form $u_{i} u_{i+1} u_{j+1} u_{j} u_{i}$ and $i$ is odd, then $2 \ell=n$. This implies that $\ell=n / 2$ and since the chord length must be odd, $n \equiv 2(\bmod 4)$ must hold. If $i$ is even, the same conclusion arises. 
If the 4-cycle has the form $u_{i} u_{i+1} u_{j} u_{j+1} u_{i}$ and $i$ is odd, then $\ell=(n+2) / 2$ which forces $n \equiv 0(\bmod 4)$ because $\ell$ is odd. On the other hand, if $i$ is even, then $\ell=(n-2) / 2$ and again $n \equiv 0(\bmod 4)$ must hold. This completes the proof.

The preceding proposition tells us exactly when a degenerate honeycomb toroidal graph has girth 4 .

Corollary 4.3. The degenerate honeycomb toridal graph $\operatorname{HTG}(1, n, \ell)$ is even edge-pancyclic if and only if one of the following holds: $\ell=3, \ell=$ $n-3, \ell=n / 2$ and $n \equiv 2(\bmod 4$, or $(1, n, \ell) \in\{(1,12,5),(1,12,7),(1,16,7)$, $(1,16,9)$,$\} .$

Proof. In order for $\operatorname{HTG}(1, n, \ell)$ to be even edge-pancyclic, it must contain a 4-cycle so that Proposition 4.2 tells us the conditions of the corollary are necessary. It is easy to verify that $\operatorname{HTG}(1, n, 3)$ is even edge-pancyclic. The same conclusion holds for $\operatorname{HTG}(1, n, n-3)$ because it is isomorphic to $\operatorname{HTG}(1, n, 3)$.

The graph $\operatorname{HTG}(1, n, n / 2)$ is isomorphic to the circulant graph of order $n$ with connection set $\{ \pm 1, n / 2\}$, and the latter graph is even edge-pancyclic by Theorem 1.1 when $n \equiv 2(\bmod 4)$.

Because $\operatorname{HTG}(1, n,(n-2) / 2)$ and $\operatorname{HTG}(1, n,(n+2) / 2)$ are isomorphic, we concentrate on the former. It is easy to verify directly that $\operatorname{HTG}(1,12,5)$ and $\operatorname{HTG}(1,16,7)$ and are even edge-pancyclic. The proof is complete upon showing that $\operatorname{HTG}(1, n,(n-2) / 2)$ is not even edge-pancyclic when $n \geq 24$ and $n \equiv 0(\bmod 4)$.

We claim that the latter graphs contain no 8-cycles. If they contain an 8-cycle, then the cycle contains subpaths of the outer Hamilton cycle connected by chords. The only cycles with a single chord have lengths $n / 2$ and $(n+2) / 2$ both of which are greater than 8 .

If a cycle uses exactly two chords, by checking the possible lengths of the paths joining them, we see that we can obtain the small lengths 4,6 , and 10 but not 8 . If a cycle use three chords and we want to achieve length 8 , then there must be two additional vertices in the cycle. One possibility is to have a path of length 3 from the outside Hamilton cycle joining two ends of two of the chords. But then the other ends of the two chords are either next to each other or distance 5 apart. There is no way a third chord can be used to form an 8-cycle because the chords have length at least 9 (when $n=20)$.

If we have a single vertex between two chords on a cycle, then the other ends of the chords also are distance 2 from each other. It is impossible to use a third chord to form an 8-cycle. 
The last possibility is that four chords can be used to form an 8-cycle. As the chords form a matching, there can be no additional vertices involved, that is the ends of the four chords must pair up as neighbors on the outer cycle. Because of the rotational symmetries of the graph, we may assume such a subpath either is $u_{0} u_{1}$ or $u_{1} u_{2}$ are the ends of neighboring chords.

For the subpath $u_{0} u_{1}$, the chords join to $u_{(n-2) / 2}$ and $u_{(n+4) / 2}$, respectively. It is now easy to verify that there is no way of using two additional chords to get an 8-cycle. A similar argument works for the edge $u_{1} u_{2}$. This completes the proof.

Honeycomb toroidal graphs have a natural embedding on the torus so that all faces are hexagons. From this it easy to find cycles of all lengths $\ell \equiv 2(\bmod 4)$. We have seen that there are some with no 8 -cycles. It may be the case that other cycles of length $\ell \equiv 0(\bmod 4)$ are missing but we do not have a good picture of what is going on here. A nice problem is to try to get an accurate picture of which cycle lengths are not achieved for not only honeycomb toroidal graphs but Cayley graphs on generalized dihedral groups for which $S_{1}=\emptyset$.

\section{References}

[1] B. Alspach (2004). Cayley Graphs, in Topics in Algebraic Graph Theory, (L. Beineke and R. Wilson, eds.) Cambridge University Press, London, 156-178. MR2125091

[2] B. Alspach, T. Bendit, and C. Maitland (2013). Pancyclicity and Cayley graphs on abelian groups, J. Graph Theory 74 260-274. MR3105550

[3] B. Alspach and C.-Q. Zhang (1989). Hamilton cycles in cubic Cayley graphs on dihedral groups, Ars Combin. 28 101-108. MR1039136

[4] T. Araki and Y. Shibata (2002). Pancyclicity of recursive circulant graphs, Inform. Process. Lett. 81 187-190. MR1881736

[5] Z. Bogdanowicz (1996). Pancyclicity of connected circulant graphs, J. Graph Theory 22 167-174. MR1393347

[6] J. A. Bondy (1971). Pancyclic graphs I, J. Combin. Theory Ser. B 11 80-84. MR0285424

[7] J. F. Fang (2011). The edge-fault-tolerant bipancyclicity of the even $k$-ary n-cube, Computer J. 54 255-262.

[8] W. Goddard and M. Henning (2001). Pancyclicity of the prism, Discrete Math. 234 139-142. MR1826827 
[9] S.-Y. Hsieh and T.-J. Lin (2009). Panconnectivity and edge-pancyclicity of $k$-ary $n$-cubes, Networks 54 1-11. MR2547426

[10] C.-Y. Li, S.-M. Tang, and Y.-L. Wang. Pancyclicity on generalized recursive circulant graphs, Proc. 27th Workshop Combin. Mathematics and Computation Theory, 118-123.

Brian Alspach

School of Mathematical and Physical Sciences

UNIVERSITY OF NEWCASTLE

Callaghan, NSW 2308

Australia

E-mail address: brian.alspach@newcastle.edu.au

Alexander Muir

Department of Defence

Russell, ACT 2600

Australia

E-mail address: alexander.p.muir@gmail.com

ReCEIVEd 16 JANuARY 2015 